\title{
SIZE AND GRAIN-BOUNDARY EFFECTS IN THE ELECTRICAL CONDUCTIVITY OF THIN MONOCRYSTALLINE FILMS
}

\author{
C. R. TELLIER \\ Université de Nancy 1, Laboratoire d'Electronique, Case Officielle No. 140, \\ 54037 Nancy Cedex, France
}

(Received September 29, 1977)

\begin{abstract}
By assuming that the scattering processes from other sources than grain-boundaries can be described by a single relaxation time $\tau^{*}$ and then by solving a Boltzmann equation in which grain-boundary scattering is accounted for, we have obtained an analytical expression for the thin monocrystalline film conductivity in terms of the reduced thickness $k$ and the grain-boundary reflection coefficient $r$. Numerical tables are given to show the agreement of the above expression with the Mayadas-Shatzkes expression.
\end{abstract}

\section{INTRODUCTION}

Several investigators ${ }^{1-8}$ have reported both experimental results ${ }^{1-5}$ and theoretical expressions ${ }^{6-8}$ for the thickness dependence of the electrical resistivity of polycrystalline and monocrystalline metallic films on the basis of the Mayadas-Shatzkes model (M-S model). ${ }^{6,7}$ However this theory leads to a rather complicated expression which involves the use of a computer to obtain numerical solutions. The purpose of this paper is to derive an analytical expression of the thin monocrystalline film resistivity by assuming that in such films the transition probability of a carrier in state $\mathrm{k}$ being scattered to a state $\mathbf{k}^{\prime}$ by all types of scatterers other than the grain-boundaries, can be expressed in term of a relaxation time $\tau^{*}$.

Let us recall that in monocrystalline metallic films, carriers suffer scattering from phonons and point defects (background scattering), external surfaces and grain-boundaries and that, in the M-S model the operative grain-boundaries can be represented by a series of randomly spaced partially reflecting planes, perpendicular to the electric field $\mathbf{E}_{\boldsymbol{x}}$ whose normal lies in the substrate surface.

Mayadas and Shatzkes have solved the general problem by following the lines of the FuchsSondheimer (F-S) calculation; $;{ }^{9}, 10$ in particular they have introduced into the Boltzmann equation an effective relaxation time $\tau_{\text {eff }}$ which takes into account the background and grain-boundaries scattering processes occuring simultaneously within the film.
Then the total film conductivity $\sigma_{F}$ is expressed as;

$$
\sigma_{F}=\sigma_{o}\left[f(\alpha)-A^{*}\right]
$$

where $\sigma_{o}$ is the bulk conductivity (i.e. the conductivity of an infinitely thick monocrystalline film) and where $A^{*}$ is given by

$$
\begin{gathered}
A^{*}=\frac{6(1-p)}{\pi k} \int_{0}^{\pi / 2} d \phi \int_{1}^{\infty} d t \frac{\cos ^{2} \phi}{H^{2}(t, \phi)}\left(\frac{1}{t^{3}}-\frac{1}{t^{5}}\right) . \\
\frac{1-\exp -k t H(t, \phi)}{1-p \exp -k t H(t, \phi)} \\
H(t, \phi)=1+\frac{\alpha}{\cos \phi}\left(1-\frac{1}{t^{2}}\right)^{-1 / 2}
\end{gathered}
$$

and where $f(\alpha)$ is given by

$$
f(\alpha)=\frac{\sigma_{g}}{\sigma_{o}}=1-\frac{3}{2} \alpha+3 \alpha^{2}-3 \alpha^{3} \ln \left(1+\frac{1}{\alpha}\right)
$$

$k$ is the ratio between film thickness $a$ and bulk mean free path $\ell_{o}$ (i.e. the reduced thickness) and $\sigma_{g}$ is the conductivity as modified by grain-boundary scattering.

The parameter $\alpha$ is related to the bulk mean free path $\ell_{o}$, average grain diameter $a_{g}$ and "grainboundary reflection coefficient" $r$ by Eq. (5) 


$$
\alpha=\frac{\ell_{o}}{a_{g}} \frac{r}{1-r}
$$

In monocrystalline films the average grain diameter $a_{g}$ is found to be equal to the film thickness $a$; from a simplistic point of view the contributions of grain-boundaries or external surfaces to the total film resistivity become comparable at this point. Consequently, in this paper we propose an analysis which consists of superimposing the grain boundaries effect and the F-S effect.

\section{EXTERNAL SURFACES SCATTERING (F-S EFFECT)}

The F-S theory is based upon the assumptions of a free electron, isotropic bulk relaxation time $\tau_{o}$ and a boundary condition for electronic distribution function which states that a fraction $p$ of electrons is specularly reflected from both surfaces of the film, ${ }^{10}$ the remainder being diffusely scattered. The film conductivity $\sigma_{F}^{*}$ (without grain boundaries effect) is given by

$$
\sigma_{F}^{*}=\sigma_{o}[1-A(k)]
$$

where $A(k)$ is a function of the reduced thickness $k$ which is generally expressed ${ }^{11}$ as

$$
A(k)=\frac{3}{2 k}(1-p) \int_{1}^{\infty}\left(\frac{1}{t^{3}}-\frac{1}{t^{5}}\right) \frac{1-e^{-k t}}{1-p \cdot e^{-k t}} d t
$$

The $1-A(k)$ function has been tabulated for different values of the specularity parameter $p$ by several authors. ${ }^{10,12,13}$

It is assumed that we may define a total relaxation time $\tau^{*}$ for the simultaneous background and external surface scattering effects so that the film conductivity $\sigma_{F}$ can be rewritten in the form

$$
\sigma_{F}^{*}=\frac{n e^{2}}{m} \tau^{*}
$$

Thus from Eq. (6) we derive

$$
\tau^{*}=\tau_{o}[1-A(k)]
$$

\section{TOTAL FILM CONDUCTIVITY}

To find the total film conductivity in the presence of grain boundaries we follow the lines of the M-S analysis $^{6,7}$ and we suppose that the grain-boundaries are represented by $N$ planes whose positions $x$ are distributed according to a Gaussian probability distribution with a standard deviation $s$.

With the assumption that the scattering from other sources (i.e. phonons, point defects and external surfaces) can be described by a relaxation time constant $\tau^{*}$, the Boltzmann equation takes the form

$$
\begin{aligned}
e E_{x} v_{x} \frac{\partial f_{o}(\mathbf{k})}{\partial \epsilon}=\int p_{\mathbf{k}}^{\mathbf{k}^{\prime}}[\Phi(\mathbf{k})- & \\
& \left.\Phi\left(\mathbf{k}^{\prime}\right)\right] d \mathbf{k}+\frac{\Phi(\mathbf{k})}{\tau^{*}}
\end{aligned}
$$

where $p_{\mathbf{k}}^{\mathbf{k}^{\prime}}$ is the transition probability for an electron in state $\mathbf{k}$ to be scattered into state $\mathbf{k}^{\prime}$ by the grainboundaries. The quantity $\Phi(\mathbf{k})$, given by $\Phi(\mathbf{k})=f(\mathbf{k})-$ $f_{o}(\mathbf{k})$ measures the deviation of the distribution function $f(\mathbf{k})$ from its equilibrium value $f_{\Omega}(\mathbf{k})$.

After a treatment identical to the M-S calculation which consists in considering the potential $V(x)$ lying at the position $x_{n}$ of the $n t h$ plane as a perturbation on the free electron Hamiltonian, the transition probability $p_{\mathbf{k}}^{\mathbf{k}^{\prime}}$ becomes: ${ }^{7}$

$$
p_{\mathbf{k}}^{\mathbf{k}^{\prime}}=F\left(\left|k_{x}\right|\right) \delta\left(k_{t}-k_{t^{\prime}}\right) \delta\left(k_{x}+k_{x^{\prime}}\right)
$$

where $k_{t}$ is the component of $\mathbf{k}$ in the $y, z$ plane and

$$
\begin{aligned}
& F\left(\left|k_{x}\right|\right)=\frac{\alpha}{2 \tau_{o}} \frac{k_{F}}{\left|k_{x}\right|} \\
& \frac{1-e^{-4 k_{x}^{2} s^{2}}}{1+e^{-4 k_{x}^{2} s^{2}}-2 e^{-k_{x}^{2} s^{2} \cdot \cos 2 k_{x} \mathrm{~d}}}
\end{aligned}
$$

Note that the bulk relaxation time $\tau_{o}$ has been artificially introduçed in Eq. (12) in a such way that the parameter $\alpha$ is related to the bulk mean free path (Eq. 5).

Under the assumptions that the interplanar spacing $d$ can be identified with the average grain diameter $a_{g}$ and that $k_{F}^{2} s^{2} \gg 1$, the function $F\left(\left|k_{x}\right|\right)$ reduces to

$$
F\left(\left|k_{x}\right|\right)=\frac{\alpha}{2 \tau_{o}} \frac{k_{F}}{\left|k_{x}\right|}=\frac{\alpha}{2 \tau_{o}|\cos \theta|}
$$

where $k_{x}$ is the $x$-component of the Fermi wave vector $k_{F}$. 
The Boltzmann equation is now: ${ }^{7}$

$$
\Phi(\mathbf{k})=\tau_{\mathrm{eff}} e E_{x} v_{x} \frac{\partial f_{o}}{\partial \epsilon}
$$

where

$$
\frac{1}{\tau_{\text {eff }}}=\frac{1}{\tau^{*}}+2 F\left(\left|k_{x}\right|\right)
$$

and $\epsilon$ the electron energy.

And the total film conductivity $\sigma_{F}$ is found to be

$$
\begin{aligned}
\sigma_{F}=\frac{e^{2}}{4 \pi^{3}} \int \frac{\tau_{\text {eff }} v_{x}^{2}}{\left|\operatorname{grad}_{k} \epsilon\right|} & d S_{\text {Fermi }}= \\
& \frac{3}{2} \frac{\sigma_{o}}{\tau_{o}} \int_{0}^{\pi} \tau_{\text {eff }} \cos ^{2} \theta \sin \theta d \theta
\end{aligned}
$$

and can be rewritten in the form

$$
\begin{aligned}
\sigma_{F}=\frac{3}{2} \frac{\sigma_{o}}{\tau_{o}} \int_{0}^{\pi} \cos ^{2} \theta \cdot \tau_{o} \cdot \\
\frac{1}{\frac{1}{1-A(k)}+\frac{\alpha}{|\cos \theta|}} \sin \theta d \theta
\end{aligned}
$$

that leads to

$$
\sigma_{F} / \sigma_{o}=[1-A(k)] \cdot f[B(k)]
$$

where

$$
\begin{aligned}
& f[B(k)]=1-\frac{3}{2} B(k)+ 3 B^{2}(k)- \\
& 3 B^{3}(k) \ln \left[1+\frac{1}{B(k)}\right]
\end{aligned}
$$

and

$$
B(k)=\alpha[1-A(k)]
$$

In the case of monocrystalline films, the function $B(k)$ depends on the reduced thickness $k$ and the grain-boundary reflection coefficient $r$. As the $1-A(k)$ function has been intensively tabulated earlier, the total film conductivity $\sigma_{F}$ from the analytical expression Eq. (18) can be evaluated without using a digital computer as previously reported by several authors. ${ }^{7,14}$

\section{DISCUSSION}

Let us note that the new formulation proposed (Eq. 18) satisfies essential qualitative physical requirements e.g.

1) When the surface scattering is entirely specular (i.e. $p=1$ ) Eq. (18) can be rewritten in the form

$$
\left.\sigma_{F} / \sigma_{a}\right]_{p=1}=1-\frac{3}{2} \alpha+3 \alpha^{2}-3 \alpha^{3} \ln \left(1+\frac{1}{\alpha}\right)
$$

and the conductivity $\sigma_{F}$ coincides with the grainboundary conductivity $\sigma_{g}$ (Eq. 4 ).

2) When the grain-boundary reflection coefficient $r$ approaches zero, Eq. (18) reduces to

$$
\left.\sigma_{F} / \sigma_{o}\right]_{r \rightarrow o}=1-A(k)
$$

and the value of the total film conductivity becomes identical to that of the F-S film conductivity $\sigma_{F}^{*}$ as expected.

In the case of monocrystalline films, numerical values of the resistivity ratio $\rho_{F} / \rho_{o}$ (Eq. 18) have been calculated for different values of the specularity parameter $p$ and grain-boundary reflection coefficient $r$; the results of the relaxation time method (Eq. 18) are compared with those of the M-S theory (as tabulated by Mola and Heras, ${ }^{14}$ Eq. 1).

Tables I and II show that, the greater the values of the parameters $r$ and $p$, the larger the range of applicability of Eq. 18. For example, in the case of diffuse scattering on external surfaces $(p=0)$ we obtain a deviation less than $3 \%$ for $k$ between 0.2 and 10 with $r=0.1$ and for $k$ between 0.06 and 10 with $r=0.62$.

From examination of table II it follows that the percentage deviations from the $\mathrm{M}-\mathrm{S}$ function decrease with increasing values of the specularity parameter $p$.

We have previously proposed an approximate expression of the resistivity ratio $\rho_{F} / \rho_{o}$ that deviates by less than $6 \%$ in the 0.01 to $2 k$-range, 0 to $0.5 p$-range and 0.1 to $0.62 r$-range; $;^{21}$ this expression introduced a function $m(r)$ which has been tabulated for different values of the grainboundary reflection coefficient $r(0.1 \leqslant r \leqslant 0.62)$.

Recently, Chaudhuri and $\mathrm{Pal}^{22}$ have analyzed their experimental data in the light of the M-S theory and have obtained a value of the grainboundary reflection coefficient $r$ equal to 0.005 . When the reflection coefficient $r$ approaches zero 
the function $m(r)$ is not easy to evaluate; on the contrary, in the present model, the analytical Eq. 19 allows the calculation of the thin monocrystalline film resistivity, even for very low values of $r$, without any tabulation.

Hence, we will attempt, in a future paper, to derive from Eq. 19 an analytical expression of the thin monocrystalline film t.c.r which is valid in a large $r$-range and reduces to the F-S equation when $r$ becomes equal to zero.

\section{CONCLUSION}

It is now well established ${ }^{4,15-20}$ that thin metal films thicker than $100 \AA$ (i.e $k$ generally greater than 0.5 at room temperature) may be regarded as continuous; furthermore in the range $0.5 \leq k \leq 10 \mathrm{Eq} .18$ deviates by only $3 \%$ in the case of diffuse scattering on external surfaces and by only $1 \%$ when a fraction $p$ of electrons is specularly scattered from external surfaces $(p=0.5)$. It thus appears that the procedure

\section{TABLE I}

Thin monocrystalline films: comparison of the values of the resistivity ratio $\rho_{F} / \rho_{O}$ as given by the equations indicated for the case of diffuse scattering on external surfaces: $p=0$.

\begin{tabular}{|c|c|c|c|c|c|c|c|c|}
\hline$k$ & $\begin{array}{l}r=0.1 \\
\text { Equation } 18\end{array}$ & Equation 1 & $\begin{array}{l}r=0.22 \\
\text { Equation } 18\end{array}$ & Equation 1 & $\begin{array}{l}r=0.42 \\
\text { Equation } 18\end{array}$ & Equation 1 & $\begin{array}{l}r=0.62 \\
\text { Equation } 18\end{array}$ & Equation 1 \\
\hline 0.01 & 41.9005 & 54.8700 & 64.8423 & 81.0040 & 124.0639 & 139.7323 & 245.1942 & 257.4954 \\
\hline 0.02 & 23.1831 & 28.1572 & 34.7980 & 41.0842 & 64.4468 & 70.3962 & 125.5034 & 129.2758 \\
\hline 0.04 & 13.0317 & 14.7706 & 18.8605 & 21.1111 & 33.7082 & 35.7139 & 64.0170 & 65.1412 \\
\hline 0.06 & 9.4225 & 10.2924 & 13.3185 & 14.4523 & 23.2293 & 24.1581 & 43.4434 & 43.7736 \\
\hline 0.08 & 7.5376 & 8.0442 & 10.4659 & 11.1224 & 17.9047 & 18.3841 & 33.0730 & 33.0978 \\
\hline 0.1 & 6.3724 & 6.6870 & 8.7170 & 9.1207 & 14.6755 & 14.9164 & 26.8126 & 26.6870 \\
\hline 0.2 & 4.0303 & 3.9367 & 5.2133 & 5.1055 & 8.2065 & 7.9802 & 14.2861 & 13.8682 \\
\hline 0.4 & 2.5310 & 2.5127 & 3.1272 & 3.0775 & 4.6318 & 4.5070 & 7.6790 & 7.4578 \\
\hline 0.6 & 2.0391 & 2.0186 & 2.4393 & 2.3917 & 3.4473 & 3.3459 & 5.4839 & 5.3194 \\
\hline 0.8 & 1.7829 & 1.7652 & 2.0844 & 2.0451 & 2.8437 & 2.7638 & 4.3748 & 4.2492 \\
\hline 1 & 1.4624 & 1.5107 & 1.8673 & 1.8356 & 2.4772 & 2.4137 & 3.7049 & 3.6064 \\
\hline 2 & 1.3028 & 1.2972 & 1.4257 & 1.4134 & 1.7352 & 1.7108 & 2.3555 & 2.3174 \\
\hline 4 & 1.1444 & 1.1430 & 1.2067 & 1.2034 & 1.3641 & 1.3574 & 1.6792 & 1.6679 \\
\hline 6 & 1.0942 & 1.0936 & 1.1360 & 1.1347 & 1.2421 & 1.2391 & 1.4544 & 1.4492 \\
\hline 8 & 1.0698 & 1.0696 & 1.1013 & 1.1006 & 1.1814 & 1.1798 & 1.3420 & 1.3390 \\
\hline 10 & 1.0555 & 1.0554 & 1.0808 & 1.0803 & 1.1451 & 1.1441 & 1.2744 & 1.2725 \\
\hline
\end{tabular}

TABLE II

Thin monocrystalline films: comparison of the values of the resistivity ratio $\rho_{F} / \rho_{O}$ as given by the equations indicated for the case of partially specular scattering on external surfaces: $\rho=0.5$

\begin{tabular}{|c|c|c|c|c|c|c|c|c|}
\hline$k$ & $\begin{array}{l}r=0.1 \\
\text { Equation } 18\end{array}$ & Equation 1 & $\begin{array}{l}r=0.22 \\
\text { Equation } 18\end{array}$ & Equation 1 & $\begin{array}{l}r=0.42 \\
\text { Equation } 18\end{array}$ & Equation 1 & $\begin{array}{l}r=0.62 \\
\text { Equation } 18\end{array}$ & Equation 1 \\
\hline 0.01 & 27.1537 & 32.8003 & 50.0748 & 57.0046 & 109.0969 & 116.2411 & 230.1133 & 236.6560 \\
\hline 0.02 & 14.8837 & 17.0153 & 26.3670 & 29.0588 & 59.3451 & 58.6566 & 115.7219 & 118.8614 \\
\hline 0.04 & 8.4082 & 9.1070 & 14.1644 & 15.0774 & 28.9403 & 29.8525 & 58.8594 & 59.9413 \\
\hline 0.06 & 6.1314 & 6.4629 & 9.9810 & 10.4166 & 19.7834 & 20.2553 & 40.0203 & 40.3109 \\
\hline 0.08 & 4.9575 & 5.1364 & 7.8484 & 8.0863 & 15.2473 & 15.4599 & 30.3863 & 30.5030 \\
\hline 0.1 & 4.2325 & 4.3361 & 6.5495 & 6.6857 & 12.4727 & 12.5799 & 24.5863 & 24.6133 \\
\hline 0.2 & 2.7148 & 2.7176 & 3.8821 & 3.8778 & 6.8535 & 6.8190 & 12.5799 & 12.8364 \\
\hline 0.4 & 1.8967 & 1.8846 & 2.4870 & 2.4619 & 3.9817 & 3.9338 & 6.9851 & 6.9463 \\
\hline 0.6 & 1.6079 & 1.5978 & 2.0047 & 1.9840 & 3.0066 & 2.9687 & 5.0368 & 4.9810 \\
\hline 0.8 & 1.4591 & 1.4513 & 1.7587 & 1.7427 & 2.5138 & 2.4844 & 4.0402 & 3.9971 \\
\hline 1 & 1.3683 & 1.3622 & 1.6093 & 1.5967 & 2.2160 & 2.1928 & 3.4401 & 3.4060 \\
\hline 2 & 1.1896 & 1.1811 & 1.3047 & 1.3011 & 1.6143 & 1.6053 & 2.2261 & 2.2194 \\
\hline 4 & 1.0903 & 1.0898 & 1.1526 & 1.1513 & 1.3098 & 1.3071 & 1.6209 & 1.6199 \\
\hline 6 & 1.0531 & 1.0596 & 1.1016 & 1.1010 & 1.2075 & 1.2063 & 1.4197 & 1.4175 \\
\hline 8 & 1.0446 & 1.0446 & 1.0761 & 1.0759 & 1.1561 & 1.1555 & 1.3166 & 1.3154 \\
\hline 10 & 1.0357 & 1.0356 & 1.0609 & 1.0607 & 1.1253 & 1.1243 & 1.2545 & 1.2536 \\
\hline
\end{tabular}


proposed for analyzing the monocrystalline film resistivity is suitable and we obtain a simple expression which is easy to evaluate over large ranges of the $r$ and $p$ parameters and allows a direct comparison of the theoretical results with experimental data.

\section{REFERENCES}

1. E. E. Mola, J. Borrajo and J. M. Heras, Surf. Sci., 34 (1973) 561-570.

2. B. Singh, C. C. Ling and N. A. Surplice, Thin Solid Films, 23 (1974) S 50.

3. G. J. Van Gurp, J. Appl. Phys., 46 (1975) 1922.

4. C. R. Tellier and A. J. Tosser, Thin Solid Films, 37 (1976) 204.

5. A. K. Pal and S. Chaudhuri, J. Mat. Sci., 11 (1976) 872.

6. A. F. Mayadas, M. Shatzkes and J. F. Janak, Appl. Phys. Lett., 14 (1969) 345.

7. A. F. Mayadas and M. Shatzkes, Phys. Rev. B., 1 (1970) 1382.
8. C. R. Tellier and A. J. Tosser, Thin Solid Films, 33 (1976) L 19.

9. K. Fuchs, Proc. Cambridge Phil. Soc., 34 (1938), 100.

10. E. H. Sondheimer, Advan. Phys., 1 (1952) 1.

11. K. L. Chopra, Thin Film Phenomena.

12. G. Brandli and P. Cotti, Helv. Phys. Acta., 38 (1965) 801.

13. S. B. Soffer, J. Appl. Phys., 36 (1965) 3947.

14. E. E. Mola and J. M. Heras, Electrocomp. Sci. and Technol., 1 (1974) 77.

15. K. L. Chopra, L. C. Bobb and M. H. Francombe, J. Appl. Phys., 34 (1963) 1699.

16. B. Laville-Saint-Martin, Thin Solid Films, 6 (1970) 359.

17. C. Reale, Czech. J. Phys. B., 21 (1971) 662.

18. J. P. Chauvineau, P. Croce, G. Devant and M. F. Verhaeghe, J. Vac. Sci. and Technol., 6 (1969) 776.

19. M. Adamov, B. Perovic and T. Nenadovic, Thin Solid Films, 24 (1974) 89.

20. R. Suri, A. P. Thakoor and K. L. Chopra, J. Appl. Phys., 46 (1975) 2574.

21. C. R. Tellier and A. J. Tosser, Electrocomp. Sci. and Technol., 3 (1976) 165.

22. S. Chaudhuri and A. K. Pal, J. Appl. Phys., 48 (1977) 3455. 

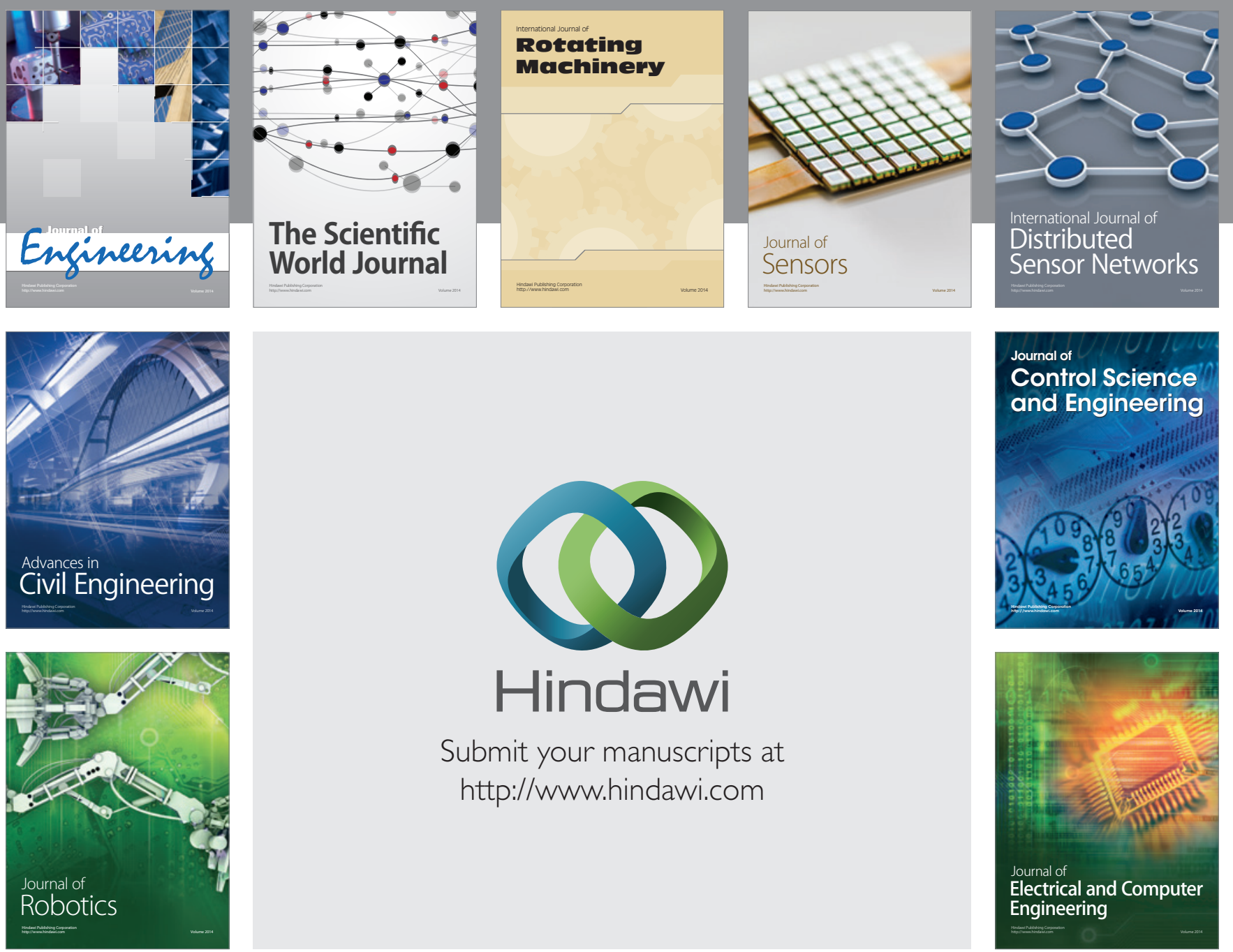

Submit your manuscripts at

http://www.hindawi.com
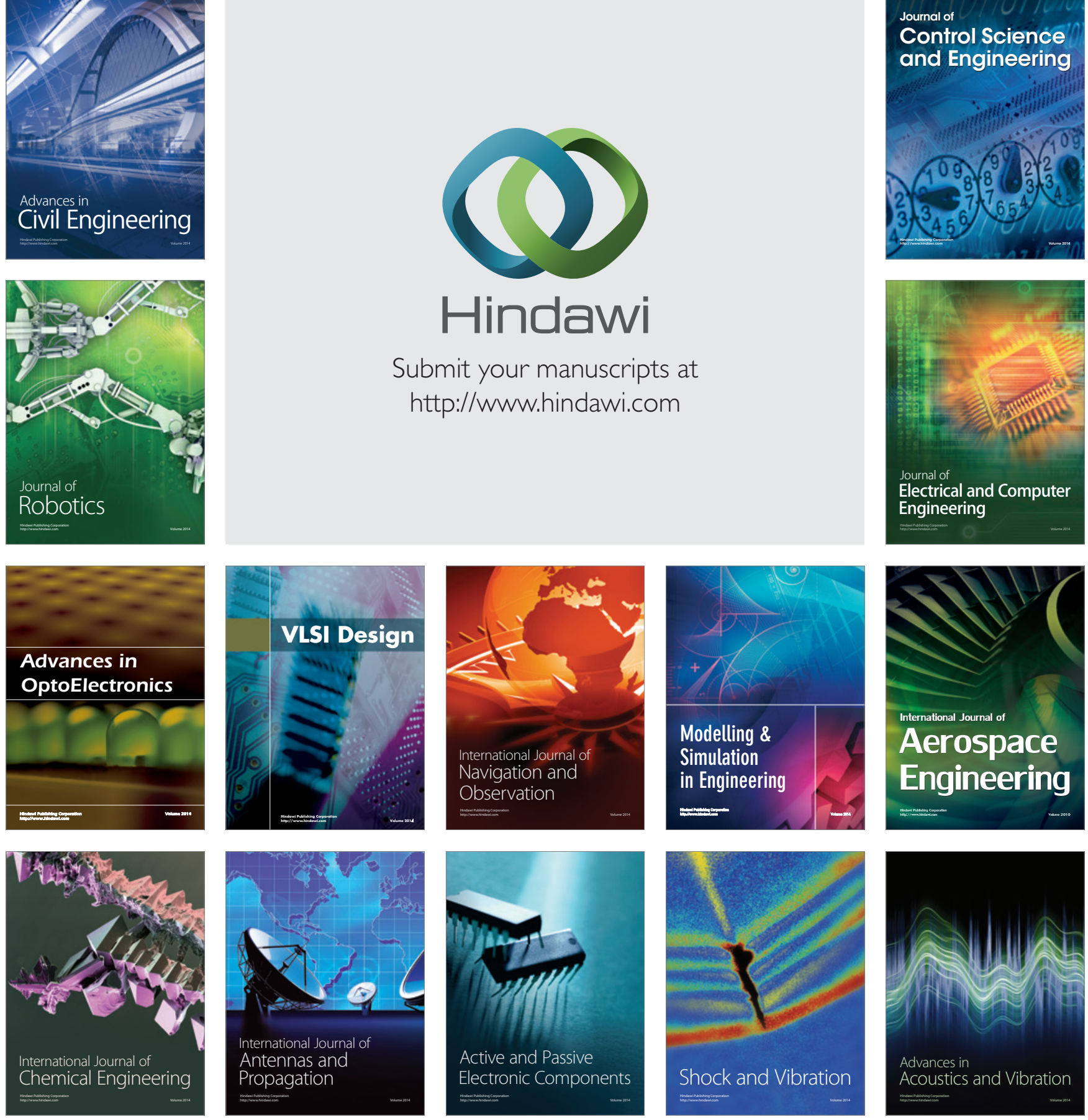